\title{
Comparison of perioperative complications following posterior column osteotomy versus posterior-based 3-column osteotomy for correction of rigid cervicothoracic deformity: a single-surgeon series of 95 consecutive cases
}

\author{
Darryl Lau, MD, ${ }^{1}$ Vedat Deviren, MD, ${ }^{2}$ Rushikesh S. Joshi, BS, ${ }^{1}$ and Christopher P. Ames, MD1 \\ Departments of ${ }^{1}$ Neurological Surgery and ${ }^{2}$ Orthopedic Surgery, University of California, San Francisco, California
}

\begin{abstract}
OBJECTIVE The correction of severe cervicothoracic sagittal deformities can be very challenging and can be associated with significant morbidity. Often, soft-tissue releases and osteotomies are warranted to achieve the desired correction. There is a paucity of studies that examine the difference in morbidity and complication profiles for Smith-Petersen osteotomy (SPO) versus 3-column osteotomy (3CO) for cervical deformity correction.
\end{abstract}

METHODS A retrospective comparison of complication profiles between posterior-based SPO (Ames grade 2 SPO) and $3 \mathrm{CO}$ (Ames grade 5 opening wedge osteotomy and Ames grade 6 closing wedge osteotomy) was performed by examining a single-surgeon experience from 2011 to 2018. Patients of interest were individuals who had a cervical sagittal vertical axis (cSVA) $>4 \mathrm{~cm}$ and/or cervical kyphosis $>20^{\circ}$ and who underwent corrective surgery for cervical deformity. Multivariate analysis was utilized.

RESULTS A total of 95 patients were included: 49 who underwent $3 \mathrm{CO}$ and 46 who underwent SPO. Twelve of the SPO patients underwent an anterior release procedure. The patients' mean age was 63.2 years, and $60.0 \%$ of the patients were female. All preoperative radiographic parameters showed significant correction postoperatively: cSVA $(6.2 \mathrm{~cm}$ vs $4.5 \mathrm{~cm}$ [preoperative vs postoperative values], $p<0.001)$, cervical lordosis $\left(6.8^{\circ}\right.$ [kyphosis] vs $\left.-7.5^{\circ}, p<0.001\right)$, and T1 slope $\left(40.9^{\circ}\right.$ and $\left.35.2^{\circ}, p=0.026\right)$. The overall complication rate was $37.9 \%$, and postoperative neurological deficits were seen in $16.8 \%$ of patients. The surgical and medical complication rates were $17.9 \%$ and $23.2 \%$, respectively. Overall, complication rates were higher in patients who underwent $3 \mathrm{CO}$ compared to those who underwent SPO, but this was not statistically significant (total complication rate $42.9 \%$ vs $32.6 \%, p=0.304$; surgical complication rate $18.4 \%$ vs $10.9 \%, p$ $=0.303$; and new neurological deficit rate $20.4 \%$ vs $13.0 \%, p=0.338$ ). Medical complication rates were similar between the two groups $(22.4 \%[3 \mathrm{CO}$ ] vs $23.9 \%$ [SPO], $p=0.866)$. Independent risk factors for surgical complications included male sex (OR 10.88, $p=0.014), c S V A>8 \mathrm{~cm}$ (OR 10.36, $p=0.037$ ), and kyphosis $>20^{\circ}(\mathrm{OR} 9.48, p=0.005)$. Combined anterior-posterior surgery was independently associated with higher odds of medical complications (OR 10.30, $p=$ 0.011 ), and preoperative kyphosis $>20^{\circ}$ was an independent risk factor for neurological deficits (OR 2.08, $p=0.011$ ).

CONCLUSIONS There was no significant difference in complication rates between $3 \mathrm{CO}$ and SPO for cervicothoracic deformity correction, but absolute surgical and neurological complication rates for $3 \mathrm{CO}$ were higher. A preoperative cSVA $>8 \mathrm{~cm}$ was a risk factor for surgical complications, and kyphosis $>20^{\circ}$ was a risk factor for both surgical and neurological complications. Additional studies are warranted on this topic.

https://thejns.org/doi/abs/10.3171/2020.3.SPINE191330

KEYWORDS cervical; complications; kyphosis; spinal deformity; three-column osteotomy

$\mathrm{T}$ HE health impact of symptomatic adult cervical deformity (ACD) is substantial, negatively affecting all EQ-5D domains, with disability scores comparable to blindness, emphysema, renal failure, and stroke. ${ }^{1}$ Severe cervical kyphotic deformities can result in dysphagia, difficulty maintaining horizontal gaze, gait impairment, and compensatory axial neck and interscapular pain. ${ }^{2}$ In fact, in severe cases, cervical kyphosis and sagittal imbalance account for many instances of myelopathy, even in the absence of central stenosis. ${ }^{3-6}$ Abnormal cervical sagittal vertical axis (cSVA) has been identified as an important parameter. cSVA is positively correlated with neck disability scores and negatively with SF-36 physical component scores; in particular, a cSVA $>4.0 \mathrm{~cm}$ is correlated with the

ABBREVIATIONS ACD = adult cervical deformity; $\mathrm{CL}=$ cervical lordosis; $\mathrm{cSVA}=$ cervical sagittal vertical axis; $\mathrm{PSO}=$ pedicle subtraction osteotomy; $\mathrm{SPO}=\mathrm{Smith}-\mathrm{Peter}-$ sen osteotomy; $3 \mathrm{CO}=3$-column osteotomy.

SUBMITTED November 6, 2019. ACCEPTED March 6, 2020.

INCLUDE WHEN CITING Published online May 8, 2020; DOI: 10.3171/2020.3.SPINE191330. 
greatest disability. ${ }^{7}$ Fortunately, patients who undergo surgical correction of their cervical deformity gain significant clinical benefits ${ }^{8}$ and improvement in myelopathy scores (Nurick score and modified Japanese Orthopaedic Association score) ${ }^{9}$

The correction of cervicothoracic deformities is formidable and technically challenging, especially in cases with rigid and fused deformities that require release via spinal osteotomies. A number of different surgical strategies exist for cervical deformity correction, including anterioronly, posterior-only, and combined anterior-posterior approaches. However, the optimal surgical approach depends on a number of anatomical and deformity-specific considerations. Osteotomy types that can be performed in the cervicothoracic region include anterior column osteotomy, Smith-Petersen osteotomy (SPO), and posteriorbased 3-column osteotomy (3CO) via pedicle subtraction osteotomy (PSO) or vertebral column resection. Cervical SPO has been shown to provide a mean angular correction of $10.1^{\circ}$ and a mean translational correction of $1.8 \mathrm{~cm}$ per level. Conversely, a single-level PSO is able to provide $34.5^{\circ}$ of angular correction on average, in addition to $2.5-4.5 \mathrm{~cm}$ of translational correction..$^{10}$ Therefore, the use of multilevel SPO should be able to obtain equivalent correction to a single PSO.

Cervical and upper thoracic SPO and 3CO are powerful corrective techniques, but they have been associated with high morbidity and complication rates. Complication rates are as high as $43.6 \%$, with mortality ranging from $1.3 \%$ to $6.7 \%$ and neurological complication rates ranging from $13.5 \%$ to $23.0 \% .{ }^{9,11,12}$ Combined anterior-posterior approaches are associated with the highest rates of early complications. ${ }^{12}$ Therefore, in select cases, there has been a progressive shift toward using posterior-only approaches to correct cervicothoracic deformities. There is a paucity of studies dedicated to examining the complication profile of posterior-based osteotomies in the correction of cervical deformity. Our study reviews the perioperative complications of patients who underwent either multilevel SPO or isolated $3 \mathrm{CO}$ for the correction of moderate to severe cervical deformity. A subgroup analysis was performed to compare perioperative complications between SPO and $3 \mathrm{CO}$. In addition, using multivariate analysis, we sought to identify independent risk factors associated with the risk of postoperative complication.

\section{Methods}

This study was approved by the Committee of Human Research at the University of California, San Francisco.

\section{Patients}

A consecutive cohort of adult patients (18 years of age or older) with a diagnosis of cervical or cervicothoracic deformity who underwent a posterior-based osteotomy for deformity correction performed by the senior author (C.P.A.) was retrospectively identified from the years 2011 to 2018. Both multilevel SPO and 3CO were performed throughout the study period. Inclusion criteria were a cSVA of $4.0 \mathrm{~cm}$ or greater and/or cervical kyphosis > $20^{\circ}$ treated with either SPO (Ames grade $2 \mathrm{SPO}$ ) or $3 \mathrm{CO}$
(Ames grade 5 opening wedge osteotomy and Ames grade 6 closing wedge osteotomy). ${ }^{13}$ SPO includes complete resection of the superior and inferior facet joints typically from C3 to C7. This also involves removal of the ligamentum flavum, spinous processes, and lamina (partial in cases without central stenosis). The $3 \mathrm{CO}$ technique has been previously described by the senior author. ${ }^{8}$ Patients who had a diagnosis of a recent infection, acute trauma, and/or tumor were excluded. Indications for surgery were significant dysfunction, debilitating axial neck pain, and/or neurological deficits (radiculopathy and/or myelopathy). All patients underwent open deformity correction with longsegment instrumentation. The decision to utilize multilevel SPO or 3CO was dependent on a variety of patient- and deformity-specific factors. In general, multilevel SPO was utilized in patients who have rigid deformities primarily involving the subaxial cervical spine, have the majority of anterior and middle columns unfused (open disc spaces), and/or have low to normal T1 slopes. 3COs were reserved for cases of rigid fused cervical deformities with severe sagittal deformities and/or high T1 slope (which represent upper thoracic deformities resulting in cervical deformity).

\section{Data Points}

Demographics, baseline clinical variables, and surgical details were retrospectively reviewed and recorded; they included age, sex, body mass index, smoking status, preoperative neurological deficit (normal strength vs weakness), abnormal gait, osteotomy type (SPO vs 3CO), osteotomy level, number of levels fused, approach, use of decompression, and comorbidities (psychiatric disease, hypertension, diabetes, thyroid disease, gastrointestinal tract disease, cardiac disease, pulmonary disease, hyperlipidemia, hepatic disease, and renal disease). Radiographic parameters of interest included preoperative and postoperative cSVA, cervical lordosis (CL), T1 slope, and coronal Cobb angle. All measurements were made using standing upright 3-foot radiographs. The specific methodology used to measure these cervical parameters has been described in a review article by Scheer et al. ${ }^{7}$

The primary outcomes of interest were overall perioperative complications within the 30 -day postoperative period; these were further stratified into neurological, surgical, and medical complications. A medical or surgical complication was defined as any unforeseen postoperative event that required additional medical and/or surgical intervention. A neurological complication was defined as new weakness on physical examination following cervical deformity correction. Sensory changes were not included.

\section{Statistical Analysis}

In order to assess the association between osteotomy grade and complications, descriptive statistics were used to summarize the cohort and univariate statistics were used to assess for associations between covariates and outcomes of interest. The chi-square test was used for comparison of categorical outcomes, and ANOVA was used for continuous outcomes. Multivariate analysis models were then employed to determine whether surgical parameters and changes in other covariates were indepen- 
TABLE 1. Preoperative and postoperative cervical parameters following correction of rigid cervicothoracic deformity

\begin{tabular}{lrrrrr}
\hline \multicolumn{1}{c}{ Parameter } & $\begin{array}{c}\text { Preop } \\
\text { Mean }\end{array}$ & SD & $\begin{array}{c}\text { Postop } \\
\text { Mean }\end{array}$ & SD & $\begin{array}{c}\text { P } \\
\text { Value }\end{array}$ \\
\hline Overall & & & & & \\
\hline $\mathrm{cSVA}(\mathrm{cm})$ & 6.2 & 1.7 & 4.5 & 1.7 & $<0.001$ \\
\hline $\mathrm{CL}\left({ }^{\circ}\right)$ & 6.8 & 26.7 & -7.5 & 15.1 & $<0.001$ \\
\hline T1 slope $\left(^{\circ}\right)$ & 40.9 & 20.8 & 35.2 & 12.9 & 0.026 \\
\hline Coronal Cobb angle $\left(^{\circ}\right)$ & 6.7 & 11.0 & 2.2 & 4.1 & $<0.001$ \\
\hline Low grade & & & & & \\
\hline $\mathrm{cSVA}(\mathrm{cm})$ & 5.5 & 1.6 & 4.3 & 1.9 & $<0.001$ \\
\hline $\mathrm{CL}\left(^{\circ}\right)$ & 14.0 & 28.4 & -5.9 & 12.6 & $<0.001$ \\
\hline T1 slope $\left(^{\circ}\right)$ & 31.5 & 16.3 & 33.7 & 13.4 & 0.483 \\
\hline Coronal Cobb angle $\left(^{\circ}\right)$ & 7.4 & 12.6 & 2.5 & 4.6 & 0.015 \\
\hline High grade & & & & & \\
\hline $\mathrm{CSVA}(\mathrm{cm})$ & 6.8 & 1.5 & 4.7 & 1.6 & $<0.001$ \\
\hline $\mathrm{CL}\left({ }^{\circ}\right)$ & 0.0 & 23.3 & -9.1 & 17.1 & 0.030 \\
\hline T1 slope $\left(^{\circ}\right)$ & 49.8 & 20.8 & 36.7 & 12.5 & $<0.001$ \\
\hline Coronal Cobb angle $\left(^{\circ}\right)$ & 5.9 & 9.2 & 1.9 & 3.6 & 0.007 \\
\hline
\end{tabular}

dently associated with the outcomes of interests. Logistic regression models were used for categorical outcomes, and ANOVA models were used for continuous outcomes. From the univariate analysis, all covariates with a $p$ value $<0.200$ were included in the multivariate model. Osteotomy grade was adjusted for in all multivariate models. For other univariate subgroup analysis, the chi-square test was used for categorical outcomes and ANOVA was used for continuous outcomes. A p value $<0.05$ was used as the threshold for statistical significance. All statistics were performed with the use of SAS version 9.4.

\section{Results}

A total of 95 patients were included in the study: 46 who underwent SPO and 49 who underwent $3 \mathrm{CO}$. The patients' mean age was 63.2 years, and $60.0 \%$ of patients were female. In the overall cohort, $61.1 \%$ of the patients presented with preoperative weakness and $56.8 \%$ presented with gait abnormalities. Of the 49 patients who underwent 3CO, osteotomies were performed at C7 (16.3\%), T1 (16.3\%), T2 (20.4\%), T3 (26.5\%), and T4 (20.4\%). Most patients $(87.4 \%)$ underwent posterior-only osteotomy, with $12.6 \%$ of SPO patients undergoing a combined anteriorposterior release. Twelve or more levels were instrumented in $52.6 \%$ of patients, and $81.1 \%$ of the patients underwent decompression.

Mean preoperative cervical parameters were as follows: cSVA, $6.2 \mathrm{~cm}$; CL, $6.8^{\circ}$ (kyphosis); T1 slope, $40.9^{\circ}$; and coronal Cobb angle, $6.7^{\circ}$ (Table 1). Overall, there was a significant change in all cervical parameters following deformity correction: cSVA, $4.5 \mathrm{~cm}(\mathrm{p}<0.001)$; $\mathrm{CL},-7.5^{\circ}(\mathrm{p}<0.001)$; T1 slope, $35.2^{\circ}(\mathrm{p}=0.026)$; and coronal Cobb angle, $2.2^{\circ}(\mathrm{p}<0.001)$. We further stratified preoperative and postoperative radiographic outcomes by osteotomy grade (Table 1). For both SPO and $3 \mathrm{CO}$, there were significant changes in all cervical parameters follow-
TABLE 2. Extent of correction achieved based on severity of preoperative sagittal deformity

\begin{tabular}{lrrrrr}
\hline & $\begin{array}{c}\text { No. of } \\
\text { Patients }\end{array}$ & $\%$ & $\begin{array}{c}\text { Postop } \\
\text { Change }\end{array}$ & SD & $\begin{array}{c}\text { p } \\
\text { Value }\end{array}$ \\
\hline Preop cSVA $(\mathrm{cm})$ & & & & & $<0.001$ \\
\hline $2-4$ & 6 & 6.3 & 1.4 & 1.1 & \\
\hline $4-6$ & 40 & 42.1 & 1.2 & 1.0 & \\
\hline $6-8$ & 33 & 34.7 & 2.0 & 1.3 & \\
\hline$>8$ & 16 & 16.8 & 3.7 & 2.2 & \\
\hline Preop kyphosis $\left(^{\circ}\right)$ & & & & & $<0.001$ \\
\hline$\leq 20$ & 69 & 72.6 & 11.8 & 8.6 & \\
\hline$>20$ & 26 & 27.4 & 38.4 & 23.5 & \\
\hline
\end{tabular}

ing deformity correction, except for T1 slope. Patients who underwent SPO did not have a significant change in T1 slope. The magnitude of angular and translational correction achieved based on cSVA and kyphosis severity was also tabulated (Table 2). There was a significant difference between the magnitude of correction when considering deformity severity. A greater preoperative cSVA was associated with greater translational correction $(\mathrm{p}<0.001)$ : 2-4 $\mathrm{cm}$ of cSVA (1.4-cm correction), $4-6 \mathrm{~cm}$ of cSVA (1.2- $\mathrm{cm}$ correction), $6-8 \mathrm{~cm}$ of cSVA (2.0-cm correction), and $>8 \mathrm{~cm}$ of cSVA $(3.7-\mathrm{cm}$ correction). In patients with preoperative kyphosis $>20^{\circ}$, we observed $38.4^{\circ}$ of correction compared to $11.8^{\circ}$ in patients with a smaller kyphotic deformity $(\mathrm{p}<0.001)$ (Table 2$)$.

Table 3 compares the demographics, characteristics, and surgical details between patients who underwent SPO and patients who underwent $3 \mathrm{CO}$ for cervical deformity correction. Demographics and comorbidities were similar between the groups. However, $3 \mathrm{CO}$ patients had significantly higher preoperative cSVA values (mean 6.8 vs 5.5 $\mathrm{cm}, \mathrm{p}<0.001$ ) and T1 slope (mean $49.8^{\circ}$ vs $31.5^{\circ}, \mathrm{p}<0.001$ ) compared to SPO patients. $3 \mathrm{CO}$ patients had a greater number of mean levels fused (mean 14.2 vs 10.9 levels, $p$ $<0.001$ ), and the rate of decompression was higher (mean $93.9 \%$ vs $67.4 \%, \mathrm{p}=0.001)$. SPO patients had significantly greater cervical kyphosis (mean $-14.0^{\circ}$ vs $0.0^{\circ}, \mathrm{p}=0.010$ ), and more SPO patients underwent anterior-posterior approaches (mean $21.7 \%$ vs $4.1 \%, \mathrm{p}=0.010$ ).

In all 95 patients, the overall complication rate was $37.9 \%$ (Table 4). Neurological complications occurred in $16.8 \%$ of patients. Surgical and medical complications occurred in $17.9 \%$ and $23.2 \%$, respectively. There were no significant differences in all complication types between SPO and $3 \mathrm{CO}$ patients. Complication rates for SPO were $32.6 \%$ overall, $13.0 \%$ neurological, $10.9 \%$ surgical, and $23.9 \%$ medical. The complication rates for $3 \mathrm{CO}$ were $42.9 \%$ overall, $20.4 \%$ neurological, $18.4 \%$ surgical, and $22.4 \%$ medical. When stratified by osteotomy level, there were significantly higher rates of neurological deficits at C7 and T1: C7 (37.5\%), T1 (37.5\%), T2 (20.0\%), $\mathrm{T} 3(15.4 \%), \mathrm{T} 4(0.0 \%)(\mathrm{p}=0.023)$. On univariate analysis, significant factors associated with neurological complications included sex $(\mathrm{p}=0.044)$, preoperative weakness ( $\mathrm{p}$ $=0.034)$, and kyphosis $(p=0.005)$. For surgical complica- 
TABLE 3. Comparison of demographics, patient characteristics, and surgical details between patients who underwent either SPO or $3 \mathrm{CO}$

\begin{tabular}{|c|c|c|c|}
\hline & SPO & $3 \mathrm{CO}$ & $p$ Value \\
\hline Age (yrs) & 65.4 & 61.1 & 0.365 \\
\hline Male sex & 34.8 & 44.9 & 0.315 \\
\hline $\mathrm{BMI}\left(\mathrm{kg} / \mathrm{m}^{2}\right)$ & 27.0 & 28.6 & 0.236 \\
\hline \multicolumn{4}{|l|}{ Comorbidities (\%) } \\
\hline Psychiatric & 26.1 & 44.9 & 0.056 \\
\hline Stroke & 8.7 & 10.5 & 0.108 \\
\hline Hypertension & 37.0 & 40.8 & 0.700 \\
\hline Diabetes & 6.5 & 14.3 & 0.218 \\
\hline Thyroid & 8.7 & 12.2 & 0.573 \\
\hline Gastrointestinal & 26.1 & 34.7 & 0.363 \\
\hline Pulmonary & 30.4 & 20.4 & 0.261 \\
\hline Cardiac & 13.0 & 16.3 & 0.652 \\
\hline Hyperlipidemia & 13.0 & 16.3 & 0.652 \\
\hline Hepatic & 8.7 & 4.1 & 0.356 \\
\hline Renal & 2.2 & 4.1 & 0.595 \\
\hline Preop weakness (\%) & 47.8 & 30.6 & 0.086 \\
\hline Abnormal gait (\%) & 56.5 & 30.6 & 0.011 \\
\hline \multicolumn{4}{|l|}{ Radiographic } \\
\hline cSVA (cm) & 5.5 & 6.8 & $<0.001$ \\
\hline $\mathrm{CL}\left({ }^{\circ}\right)$ & -14.0 & 0.0 & 0.010 \\
\hline T1 slope $\left(^{\circ}\right)$ & 31.5 & 49.8 & $<0.001$ \\
\hline Coronal Cobb angle $\left(^{\circ}\right)$ & 7.4 & 5.9 & 0.512 \\
\hline \multicolumn{4}{|l|}{ Surgical } \\
\hline Levels fused (mean no.) & 10.9 & 14.2 & $<0.001$ \\
\hline Combined anterior-posterior (\%) & 21.7 & 4.1 & 0.010 \\
\hline Laminectomy (\%) & 67.4 & 93.9 & 0.001 \\
\hline
\end{tabular}

$\mathrm{BMI}=$ body mass index.

tions, the significant factors were sex $(\mathrm{p}=0.009)$, kyphosis $(\mathrm{p}=0.007)$, and cSVA $(\mathrm{p}=0.037)$. Only approach type was associated with medical complications $(\mathrm{p}=0.018)$.

On multivariate analysis, male sex (OR 10.88, 95\% CI $1.62-73.00 ; \mathrm{p}=0.014)$, kyphosis $>20^{\circ}(\mathrm{OR} 9.48,95 \% \mathrm{CI}$ $1.97-45.68 ; \mathrm{p}=0.005)$, and $\mathrm{cSVA}>8 \mathrm{~cm}(\mathrm{OR} 10.36,95 \%$ CI $1.15-93.45 ; \mathrm{p}=0.037$ ) were independently associated with higher odds of surgical complications (Table 5). Patients with kyphosis $>20^{\circ}$ also had significantly higher odds of experiencing neurological complications relative to patients with a smaller kyphotic curvature (OR 2.08, 95\% CI 1.81-6.53; $\mathrm{p}=0.011)$. A combined anterior-posterior approach was an independent factor associated with medical complications (OR 10.30, 95\% CI 1.69-62.67; p $=0.011$.

To investigate the relationship between the magnitude of correction and the presence of complications, we compared preoperative and postoperative cSVA and kyphosis values in patients who experienced complications versus those who experienced no complications (Table 6). Patients who had a neurological or medical complication underwent significantly greater kyphosis correction $(\mathrm{p}=$ 0.041 and $p=0.028$, respectively) than patients without those complications. Patients who experienced a surgical complication underwent significantly greater correction of cSVA $(p=0.039)$ than those who did not.

\section{Illustrative Cases}

\section{Postlaminectomy Kyphosis: PSO}

A 54-year-old woman who had previously undergone cervical decompression presented with difficult holding up her head up and was found to have a severe rigid cervical deformity (postlaminectomy kyphosis) (Fig. 1A and B). CT scanning demonstrated a focal kyphotic deformity in which the C6 and C7 vertebral bodies fused together (Fig. 1C). Preoperative cervical parameters were as follows: cSVA, $9.4 \mathrm{~cm}$; $\mathrm{CL},-12^{\circ}$ (kyphosis); C2 slope, $40^{\circ}$; and T1 slope, $32^{\circ}$. The patient underwent a C6-7 PSO and C2-T10 instrumented posterior spinal fusion (Fig. 1D and E). Her postoperative parameters were as follows: cSVA, $4.7 \mathrm{~cm} ; \mathrm{CL}, 10^{\circ}$; $\mathrm{C} 2$ slope, $25^{\circ}$; and $\mathrm{T} 1$, slope $34^{\circ}$.

\section{Postlaminoplasty Kyphosis: Multiple-Level SPO}

A 61-year-old woman with a history of cervical laminoplasty presented with chin-on-chest deformity (Fig. 2A and $\mathrm{B}$ ). Her preoperative cervical parameters were as follows: cSVA, $7.7 \mathrm{~cm}$; $\mathrm{CL},-40^{\circ}$ (kyphosis); $\mathrm{C} 2$ slope, $45^{\circ}$; and $\mathrm{T} 1$ slope, $37^{\circ}$. She underwent a C3-7 SPO C2-T10 instrumented posterior spinal fusion (Fig. 2C and D). Her postoperative cervical parameters were as follows: cSVA, $5.6 \mathrm{~cm} ; \mathrm{CL}, 2^{\circ}$; $\mathrm{C} 2$ slope, $25^{\circ}$; and $\mathrm{T} 1$ slope, $34^{\circ}$.

\section{Discussion}

Severe cervical and cervicothoracic deformities are considered the most debilitating of all spinal deformities. ${ }^{1}$ Patients experience low self-esteem, poor quality of life, debilitating pain, and progressive neurological decline. ${ }^{2-6}$ Radiographic parameters such as cSVA have been shown to be strongly correlated with significant functional disability based on health-related quality-of-life questionnaires, specifically when cSVA is $>4.0 \mathrm{~cm} .{ }^{14}$ However, there are mixed results and findings as to whether regional CL correlates with clinical outcomes and postoperative pain. ${ }^{15,16}$ In fact, some studies have suggested that changes in regional CL do not correlate significantly with clinical outcomes. ${ }^{17}$ However, correction of ACD has been shown to provide significant benefits with regard to Neck Disability Index, neck pain visual analog scale scores, and SF-36 physical component summary scores. ${ }^{8}$

The first consideration in surgically managing patients with cervicothoracic deformity is to determine the primary driver of the cervical abnormality (isolated cervical vs combined cervical and cervicothoracic junction vs isolated upper thoracic). ${ }^{18}$ This determination will impact the decision of choosing osteotomy type and level. For example, patients with a high T1 slope and low C2 slope likely have an upper thoracic deformity and would benefit from an upper thoracic osteotomy for cervicothoracic deformity correction. The observed differences in preoperative cervical parameters between SPO (greater CL) and 3CO (greater cSVA and T1 slope) patients help demonstrate these concepts. Multilevel SPOs are most effective 
TABLE 4. Relationship of demographics and operative variables with perioperative complications

\begin{tabular}{|c|c|c|c|c|c|c|c|c|c|c|c|c|c|c|}
\hline & \multirow{2}{*}{$\begin{array}{c}\text { Overall } \\
\text { No. }\end{array}$} & \multirow[b]{2}{*}{$\%$} & \multicolumn{3}{|c|}{ Complications } & \multicolumn{3}{|c|}{ Neurological } & \multicolumn{3}{|c|}{ Surgical } & \multicolumn{3}{|c|}{ Medical } \\
\hline & & & No. & $\%$ & $p$ Value & No. & $\%$ & p Value & No. & $\%$ & $p$ Value & No. & $\%$ & $p$ Value \\
\hline Total & 95 & & 36 & 37.9 & & 16 & 16.8 & & 17 & 17.9 & & 22 & 23.2 & \\
\hline Age (yrs) & & & & & 0.355 & & & 0.226 & & & 0.576 & & & 0.174 \\
\hline$<55$ & 11 & 11.6 & 6 & 54.5 & & 0 & 0.0 & & 1 & 9.1 & & 5 & 45.5 & \\
\hline $55-70$ & 63 & 66.3 & 24 & 38.1 & & 13 & 20.6 & & 11 & 17.5 & & 13 & 20.6 & \\
\hline$>70$ & 21 & 22.1 & 6 & 28.6 & & 3 & 14.3 & & 2 & 9.5 & & 4 & 19.0 & \\
\hline Sex & & & & & 0.262 & & & 0.044 & & & 0.009 & & & 0.691 \\
\hline Female & 57 & 60.0 & 19 & 33.3 & & 6 & 10.5 & & 4 & 7.0 & & 14 & 24.6 & \\
\hline Male & 38 & 40.0 & 17 & 44.7 & & 10 & 26.3 & & 10 & 26.3 & & 8 & 21.1 & \\
\hline $\mathrm{BM}\left(\mathrm{kg} / \mathrm{m}^{2}\right)$ & & & & & 0.355 & & & 0.106 & & & 0.206 & & & 0.071 \\
\hline$<18.5$ & 3 & 3.2 & 0 & 0.0 & & 0 & 0.0 & & 0 & 0.0 & & 0 & 0.0 & \\
\hline $18.5-25.0$ & 27 & 28.4 & 13 & 48.1 & & 1 & 3.7 & & 1 & 3.7 & & 11 & 40.7 & \\
\hline $25.0-30$ & 35 & 36.8 & 12 & 34.3 & & 9 & 25.7 & & 7 & 20.0 & & 6 & 17.1 & \\
\hline$>30$ & 30 & 31.6 & 11 & 36.7 & & 6 & 20.0 & & 6 & 20.0 & & 5 & 16.7 & \\
\hline Smoking status & & & & & 0.582 & & & 0.438 & & & 0.745 & & & 0.702 \\
\hline Never & 45 & 47.4 & 15 & 33.3 & & 7 & 15.6 & & 6 & 13.3 & & 9 & 20.0 & \\
\hline Former & 36 & 37.9 & 16 & 44.4 & & 5 & 13.9 & & 5 & 13.9 & & 10 & 27.8 & \\
\hline Active & 14 & 14.7 & 5 & 35.7 & & 4 & 28.6 & & 3 & 21.4 & & 3 & 21.4 & \\
\hline \multicolumn{15}{|l|}{ Comorbidities } \\
\hline Psychiatric & & & & & 0.623 & & & 0.194 & & & 0.230 & & & 0.342 \\
\hline No & 61 & 64.2 & 22 & 36.1 & & 8 & 13.1 & & 7 & 11.5 & & 16 & 9.8 & \\
\hline Yes & 34 & 35.8 & 14 & 41.2 & & 8 & 23.5 & & 7 & 20.6 & & 6 & 17.6 & \\
\hline Stroke & & & & & 0.679 & & & 0.620 & & & 0.959 & & & 0.868 \\
\hline No & 81 & 85.3 & 30 & 37.0 & & 13 & 16.0 & & 12 & 14.8 & & 19 & 23.5 & \\
\hline Yes & 14 & 14.7 & 6 & 42.9 & & 3 & 21.4 & & 2 & 14.3 & & 3 & 21.4 & \\
\hline Hypertension & & 0.0 & & & 0.679 & & & 0.489 & & & 0.788 & & & 0.777 \\
\hline No & 58 & 61.1 & 23 & 39.7 & & 11 & 19.0 & & 9 & 15.5 & & 14 & 24.1 & \\
\hline Yes & 37 & 38.9 & 13 & 35.1 & & 5 & 13.5 & & 5 & 13.5 & & 8 & 21.6 & \\
\hline Diabetes & & & & & 0.404 & & & 0.778 & & & 0.788 & & & 0.588 \\
\hline No & 85 & 89.5 & 31 & 36.5 & & 14 & 16.5 & & 12 & 14.1 & & 19 & 22.4 & \\
\hline Yes & 10 & 10.5 & 5 & 50.0 & & 2 & 20.0 & & 2 & 20.0 & & 3 & 30.0 & \\
\hline Thyroid & & & & & 0.885 & & & 0.240 & & & 0.150 & & & 0.297 \\
\hline No & 85 & 89.5 & 32 & 37.6 & & 13 & 15.3 & & 11 & 12.9 & & 21 & 24.7 & \\
\hline Yes & 10 & 10.5 & 4 & 40.0 & & 3 & 30.0 & & 3 & 30.0 & & 1 & 10.0 & \\
\hline Gastrointestinal & & & & & 0.170 & & & 0.945 & & & 0.648 & & & 0.050 \\
\hline No & 66 & 69.5 & 28 & 42.4 & & 11 & 16.7 & & 9 & 13.6 & & 19 & 28.8 & \\
\hline Yes & 29 & 30.5 & 8 & 27.6 & & 5 & 17.2 & & 5 & 17.2 & & 3 & 10.3 & \\
\hline Pulmonary & & & & & 0.963 & & & 0.062 & 9 & & 0.330 & & & 0.755 \\
\hline No & 71 & 74.7 & 27 & 38.0 & & 9 & 12.7 & & 5 & 7.0 & & 17 & 23.9 & \\
\hline Yes & 24 & 25.3 & 24 & 100.0 & & 7 & 29.2 & & & 0.0 & & 5 & 20.8 & \\
\hline Cardiac & & & & & 0.856 & & & 0.782 & & & 0.959 & & & 0.755 \\
\hline No & 81 & 85.3 & 31 & 38.3 & & 14 & 17.3 & & 12 & 14.8 & & 18 & 22.2 & \\
\hline Yes & 14 & 14.7 & 5 & 35.7 & & 2 & 14.3 & & 2 & 14.3 & & 4 & 28.6 & \\
\hline Hyperlipidemia & & & & & 0.312 & & & 0.620 & & & 0.444 & & & 0.394 \\
\hline No & 81 & 85.3 & 29 & 35.8 & & 13 & 16.0 & & 11 & 13.6 & & 20 & 24.7 & \\
\hline Yes & 14 & 14.7 & 7 & 50.0 & & 3 & 21.4 & & 3 & 21.4 & & 2 & 14.3 & \\
\hline Hepatic & & & & & 0.812 & & & 0.991 & & & 0.890 & & & 0.697 \\
\hline No & 89 & 93.7 & 34 & 38.2 & & 15 & 16.9 & & 13 & 14.6 & & 21 & 23.6 & \\
\hline Yes & 6 & 6.3 & 6 & 100.0 & & 1 & 16.7 & & 1 & 16.7 & & 1 & 16.7 & \\
\hline
\end{tabular}


» CONTINUED FROM PAGE 301

TABLE 4. Relationship of demographics and operative variables with perioperative complications

\begin{tabular}{|c|c|c|c|c|c|c|c|c|c|c|c|c|c|c|}
\hline & \multirow{2}{*}{$\begin{array}{c}\text { Overall } \\
\text { No. }\end{array}$} & \multirow[b]{2}{*}{$\%$} & \multicolumn{3}{|c|}{ Complications } & \multicolumn{3}{|c|}{ Neurological } & \multicolumn{3}{|c|}{ Surgical } & \multicolumn{3}{|c|}{ Medical } \\
\hline & & & No. & $\%$ & $p$ Value & No. & $\%$ & p Value & No. & $\%$ & p Value & No. & $\%$ & $p$ Value \\
\hline \multicolumn{15}{|c|}{ Comorbidities (continued) } \\
\hline Renal & & & & & 0.869 & & & 0.428 & & & 0.356 & & & 0.334 \\
\hline No & 92 & 96.8 & 35 & 38.0 & & 16 & 17.4 & & 13 & 14.1 & & 22 & 23.9 & \\
\hline Yes & 3 & 3.2 & 1 & 33.3 & & 0 & 0.0 & & 1 & 33.3 & & 0 & 0.0 & \\
\hline Preop weakness & & & & & 0.381 & & & 0.034 & & & 0.358 & & & 0.200 \\
\hline No & 58 & 61.1 & 24 & 41.4 & & 6 & 10.3 & & 7 & 12.1 & & 16 & 27.6 & \\
\hline Yes & 37 & 38.9 & 12 & 32.4 & & 10 & 27.0 & & 7 & 18.9 & & 6 & 16.2 & \\
\hline Abnormal gait & & & & & 0.381 & & & 0.545 & & & 0.980 & & & 0.804 \\
\hline No & 54 & 56.8 & 20 & 37.0 & & 8 & 14.8 & & 8 & 14.8 & & 12 & 22.2 & \\
\hline Yes & 41 & 43.2 & 16 & 39.0 & & 8 & 19.5 & & 6 & 14.6 & & 10 & 24.4 & \\
\hline Kyphosis $\left({ }^{\circ}\right)$ & & & & & 0.381 & & & 0.005 & & & 0.007 & & & 0.991 \\
\hline$\leq 20$ & 69 & 72.6 & 24 & 34.8 & & 7 & 10.1 & & 6 & 8.7 & & 16 & 23.2 & \\
\hline$>20$ & 26 & 27.4 & 12 & 46.2 & & 9 & 34.6 & & 8 & 30.8 & & 6 & 23.1 & \\
\hline cSVA & & & & & 0.095 & & & 0.099 & & & 0.037 & & & 0.642 \\
\hline $2-4$ & 6 & 6.3 & 3 & 50.0 & & 1 & 16.7 & & 1 & 16.7 & & 2 & 33.3 & \\
\hline $4-6$ & 40 & 42.1 & 11 & 27.5 & & 4 & 10.0 & & 3 & 7.5 & & 7 & 17.5 & \\
\hline $6-8$ & 33 & 34.7 & 12 & 36.4 & & 6 & 18.2 & & 4 & 12.1 & & 8 & 24.2 & \\
\hline$>8$ & 16 & 16.8 & 10 & 62.5 & & 5 & 31.3 & & 6 & 37.5 & & 5 & 31.3 & \\
\hline Osteotomy type & & & & & 0.304 & & & 0.338 & & & 0.303 & & & 0.866 \\
\hline Low grade & 46 & 48.4 & 15 & 32.6 & & 6 & 13.0 & & 5 & 10.9 & & 11 & 23.9 & \\
\hline High grade & 49 & 51.6 & 21 & 42.9 & & 10 & 20.4 & & 9 & 18.4 & & 11 & 22.4 & \\
\hline Levels fused & & & & & 0.385 & & & 0.751 & & & 0.831 & & & 0.096 \\
\hline$<12$ & 45 & 47.4 & 15 & 33.3 & & 7 & 15.6 & & 7 & 15.6 & & 7 & 15.6 & \\
\hline$\geq 12$ & 50 & 52.6 & 21 & 42.0 & & 9 & 18.0 & & 7 & 14.0 & & 15 & 30.0 & \\
\hline Approach & & & & & 0.355 & & & 0.095 & & & 0.123 & & & 0.018 \\
\hline Posterior only & 83 & 87.4 & 30 & 36.1 & & 16 & 19.3 & & 14 & 16.9 & & 16 & 19.3 & \\
\hline $\begin{array}{l}\text { Combined } \\
\text { anterior-posterior }\end{array}$ & 12 & 12.6 & 6 & 50.0 & & 0 & 0.0 & & 0 & 0.0 & & 6 & 50.0 & \\
\hline Laminectomy & & & & & 0.525 & & & 0.982 & & & 0.630 & & & 0.079 \\
\hline No & 18 & 18.9 & 8 & 44.4 & & 3 & 16.7 & & 2 & 11.1 & & 7 & 38.9 & \\
\hline Yes & 77 & 81.1 & 28 & 36.4 & & 13 & 16.9 & & 12 & 15.6 & & 15 & 19.5 & \\
\hline
\end{tabular}

for patients with rigid subaxial cervical deformities resulting in cervical kyphosis, and 3COs are used in patients not only with rigid, fused deformities but also with severe sagittal imbalance (head translation) and high T1 slope. Based on prior studies, the ideal postoperative cSVA is $<$ $4.0 \mathrm{~cm} .{ }^{14}$ The goal for CL can be predicted by utilizing cervical mismatch measurements $\left(\mathrm{CL}=\mathrm{T} 1\right.$ slope $-16.5^{\circ}$ $\left.\pm 2^{\circ}\right) .{ }^{19}$ Surgical correction of moderate to severe cervical deformities often requires soft-tissue release and osteotomies. An anterior-only, posterior-only, or combined anterior-posterior approach can be utilized.

Posterior-based cervical osteotomies and releases are useful options for the correction of severe sagittal deformities involving the cervical spine. Traditionally, for rigid cervical kyphotic deformities, SPO is used; this was often supplemented by a controlled wedge fracture (particularly at C7) or concurrent anterior release to restore sagittal balance. ${ }^{20-22}$ A single-level SPO can offer $10.1^{\circ}$ of angular and $1.8 \mathrm{~cm}$ of translational correction on average. When supplemented with an anterior release, averages of $27.8^{\circ}$ of angular and $2.6 \mathrm{~cm}$ of translational correction can be achieved..$^{10}$ The cervical SPO is thought to offer greater controlled closure and stronger biomechanical stability, while avoiding the anterior open wedge defects found in the traditional cervical PSO ${ }^{23,24}$ However, given the smaller magnitude of correction possible with SPO, there is a growing interest in utilizing $3 \mathrm{CO}$ via PSO for cervicothoracic deformity correction. ${ }^{8,25-27}$ Cervical PSO is a technique that can offer correction for fixed coronal deformities as well. ${ }^{28}$ Recent efforts have demonstrated a push toward performing PSO in the cervicothoracic junction, mainly at $\mathrm{T} 1, \mathrm{~T} 2$, or $\mathrm{T} 3{ }^{25}$ The reasons for this are multifold: the canal diameter is larger in the lower cervical and upper thoracic spine, iatrogenic injury below the 
TABLE 5. Independent risk factors for specific perioperative complications following correction of rigid cervicothoracic deformity

\begin{tabular}{lcccc}
\hline $\begin{array}{c}\text { Independent Risk Factor } \\
\text { Surgical }\end{array}$ & OR & $\begin{array}{c}95 \% \mathrm{Cl} \\
(\mathrm{Min})\end{array}$ & $\begin{array}{c}95 \% \mathrm{Cl} \\
(\mathrm{Max})\end{array}$ & $\mathrm{p} \mathrm{Value}$ \\
\hline Sex & & & & \\
\hline Female & Ref & Ref & Ref & Ref \\
\hline Male & 10.88 & 1.62 & 73.00 & 0.014 \\
\hline Kyphosis $\left(^{\circ}\right)$ & & & & \\
\hline$\leq 20$ & Ref & Ref & Ref & Ref \\
\hline$>20$ & 9.48 & 1.97 & 45.68 & 0.005 \\
\hline cSVA & & & & \\
\hline $2-4$ & Ref & Ref & Ref & Ref \\
\hline $4-6$ & 0.79 & 0.05 & 12.49 & 0.864 \\
\hline $6-8$ & 3.71 & 0.23 & 60.68 & 0.359 \\
\hline$>8$ & 10.36 & 1.15 & 93.45 & 0.037 \\
\hline Neurological & & & & \\
\hline Kyphosis & & & & \\
\hline$\leq 20$ & Ref & Ref & Ref & Ref \\
\hline$>20$ & 2.08 & 1.81 & 6.53 & 0.011 \\
\hline Medical & & & & \\
\hline Approach & & & & \\
\hline Posterior only & Ref & Ref & Ref & Ref \\
\hline Combined anterior-posterior & 10.30 & 1.69 & 62.67 & 0.011 \\
\hline Ref & & &
\end{tabular}

Ref $=$ reference.

cervical levels can spare the upper extremities, and lower cervical or upper thoracic PSO allows for avoidance of the vertebral arteries, which enter the transverse foramen at C6. ${ }^{29-31}$ Lower cervical and upper thoracic PSO can offer cSVA correction of 2.2 to $4.5 \mathrm{~cm}$, cervical sagittal Cobb correction of $10.1^{\circ}$ to $34.5^{\circ}$, and average chin-brow vertical angle correction of $36.7^{\circ} .{ }^{8,10,25}$ According to our findings, multilevel SPO and $3 \mathrm{CO}$ are able to provide similar corrective abilities.

Multilevel SPOs and PSOs are clearly extremely powerful techniques for the correction of fixed cervical sagittal deformities but have been associated with high complication rates. There are no large studies comparing the complication profiles between multilevel SPO and $3 \mathrm{CO}$ for cervical and cervicothoracic deformity correction. In our study, comparison of multilevel SPO and 3CO did not demonstrate a statistically significant difference in radiological correction; however, $3 \mathrm{CO}$ had higher absolute rates of overall, neurological, and surgical complications by $10.3 \%, 7.4 \%$, and $7.5 \%$, respectively. These differences in complication rates are clinically significant and should be considered in surgical planning. The overall complication rate of $37.9 \%$ observed in our cohort is similar to prior reports. Smith and colleagues performed a prospective multicenter study of 78 patients undergoing correction of ACD and found that $43.6 \%$ of patients experienced at least one complication. ${ }^{25}$ In that study, the most common complications were dysphagia (11.5\%), wound infections (6.4\%), C5 nerve palsy (6.4\%), and respiratory failure (5.1\%). Compli-
TABLE 6. Relationship between the severity of sagittal deformity and complications

\begin{tabular}{ccccccccc}
\hline & \multicolumn{3}{c}{ Kyphosis Correction } & & \multicolumn{3}{c}{ cSVA Correction } \\
\cline { 2 - 3 } \cline { 6 - 7 } Complication & Mean & SD & p Value & & Mean & SD & $p$ V Value \\
\hline Complication & & & 0.041 & & & 0.213 \\
\hline No & 16.1 & 14.4 & & & 1.8 & 1.5 & \\
\hline Yes & 24.1 & 23.1 & & & 2.2 & 1.8 & \\
\hline Surgical & & & 0.345 & & & 0.039 \\
\hline No & 18.3 & 19.0 & & 1.8 & 1.6 & \\
\hline Yes & 23.4 & 15.1 & & 2.5 & 1.8 & \\
\hline Medical & & & 0.028 & & & 0.464 \\
\hline No & 16.8 & 14.6 & & 1.9 & 1.6 & \\
\hline Yes & 26.6 & 26.9 & & 2.1 & 1.7 & \\
\hline
\end{tabular}

cations were highest following combined anterior-posterior approaches $(79.3 \%){ }^{25}$ In a comprehensive literature review of 14 studies and 399 patients, mortality rates ranged from $3.1 \%$ to $6.7 \%$, and medical complication rates ranged from $3.1 \%$ to $44.4 \%$ following surgery for symptomatic cervical and cervicothoracic kyphosis. ${ }^{9}$

The most devastating complications that can occur during cervical deformity surgery result from injury to the vertebral artery, spinal cord, or nerve roots. Fortunately, vertebral artery injuries are extremely rare. The neurological complication rate in our study was $16.8 \%$, which was within the ranges of prior reports. Reported rates of postoperative neurological deficit following cervical deformity correction range from $12.8 \% \%$ to $23.4 \% .^{11,12,29}$ In our study, $3 \mathrm{CO}$ patients had higher absolute rates of neurological complications than SPO. The observed $3 \mathrm{CO}$ neurological deficit rate of $20.4 \%$ is similar to those of prior published studies. This may reflect the greater technical difficulty associated with a $3 \mathrm{CO}$ and/or larger extent of correction achieved at a single focal point (which may put patients at higher risk of dural buckling and nerve root impingement). Samudrala et al. reviewed their experience with 8 patients who underwent PSO for cervicothoracic kyphosis; 3 patients $(37.5 \%)$ had neurological deficits. Two patients had transient hand numbness and weakness, and 1 patient had right upper-extremity weakness requiring reoperation..$^{32}$ This neurological deficit rate was similar to that in the study published by Tokala et al. in which the authors reported C8 radiculopathy in $3(37.5 \%)$ of 8 patients following C7 PSO for cervicothoracic kyphosis. The $\mathrm{C} 8$ radiculopathies were transient and resolved within 4 weeks. ${ }^{33}$ In a study of 23 patients undergoing $3 \mathrm{CO}$ for cervicothoracic deformities, Smith et al. reported a postoperative neurological deficit in $17.4 \%$ of patients. ${ }^{25}$ Based on our experience as well as prior published studies, it is clear that there is a real risk of neurological complications when performing cervical deformity correction. Neuromonitoring modalities (MEP, SSEP, and EMG) are essential in minimizing injury to neural elements, but their performance measures have not been thoroughly studied for cervical deformity correction via $\mathrm{SPO}$ or $3 \mathrm{CO}$.

An important component to planning a $3 \mathrm{CO}$ for cervical deformity correction is to consider the level in which 

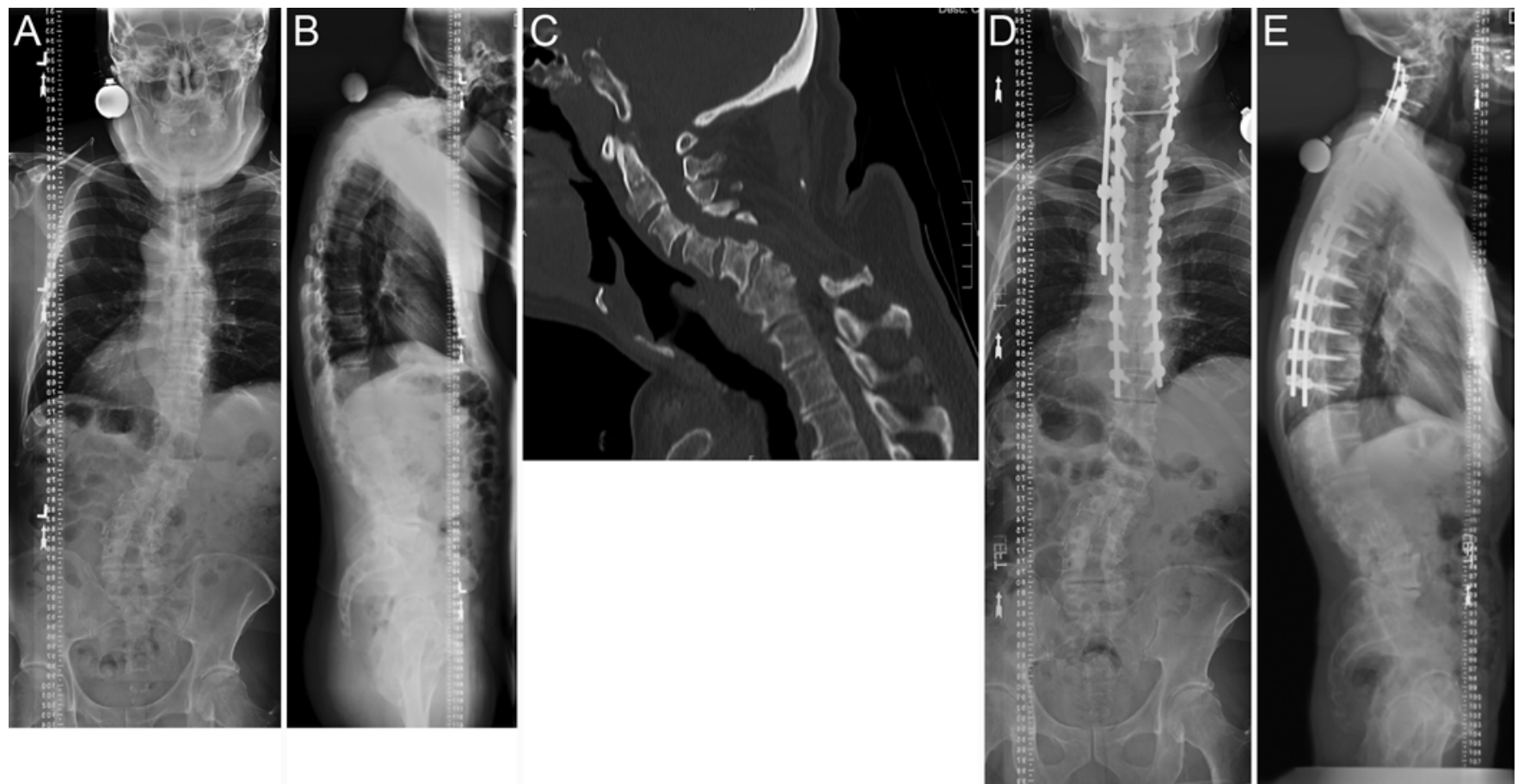

FIG. 1. Preoperative and postoperative images of a patient with severe cervicothoracic kyphosis following cervical decompression who underwent C6-7 PSO and instrumented C2-T10 posterior spinal fusion. Anterior-posterior and lateral scoliosis radiographs show severe cervicothoracic kyphosis: cSVA, $9.4 \mathrm{~cm}$; CL, $-12^{\circ}$ (kyphosis); $\mathrm{C} 2$ slope, $40^{\circ}$; and $\mathrm{T} 1$ slope, $32^{\circ}$ (A and B). Sagittal CT scan showing fused $\mathrm{C} 6$ and $\mathrm{C} 7$ segment with a rigid, focal kyphotic deformity (C). As seen on scoliosis radiographs, significant correction of the patient's cervical deformity was achieved. Postoperative parameters were CSVA, $4.7 \mathrm{~cm} ; \mathrm{CL}, 10^{\circ}$; $\mathrm{C} 2$ slope, $25^{\circ}$; and $\mathrm{T} 1$ slope, $34^{\circ}(\mathrm{D}$ and $\mathrm{E})$.
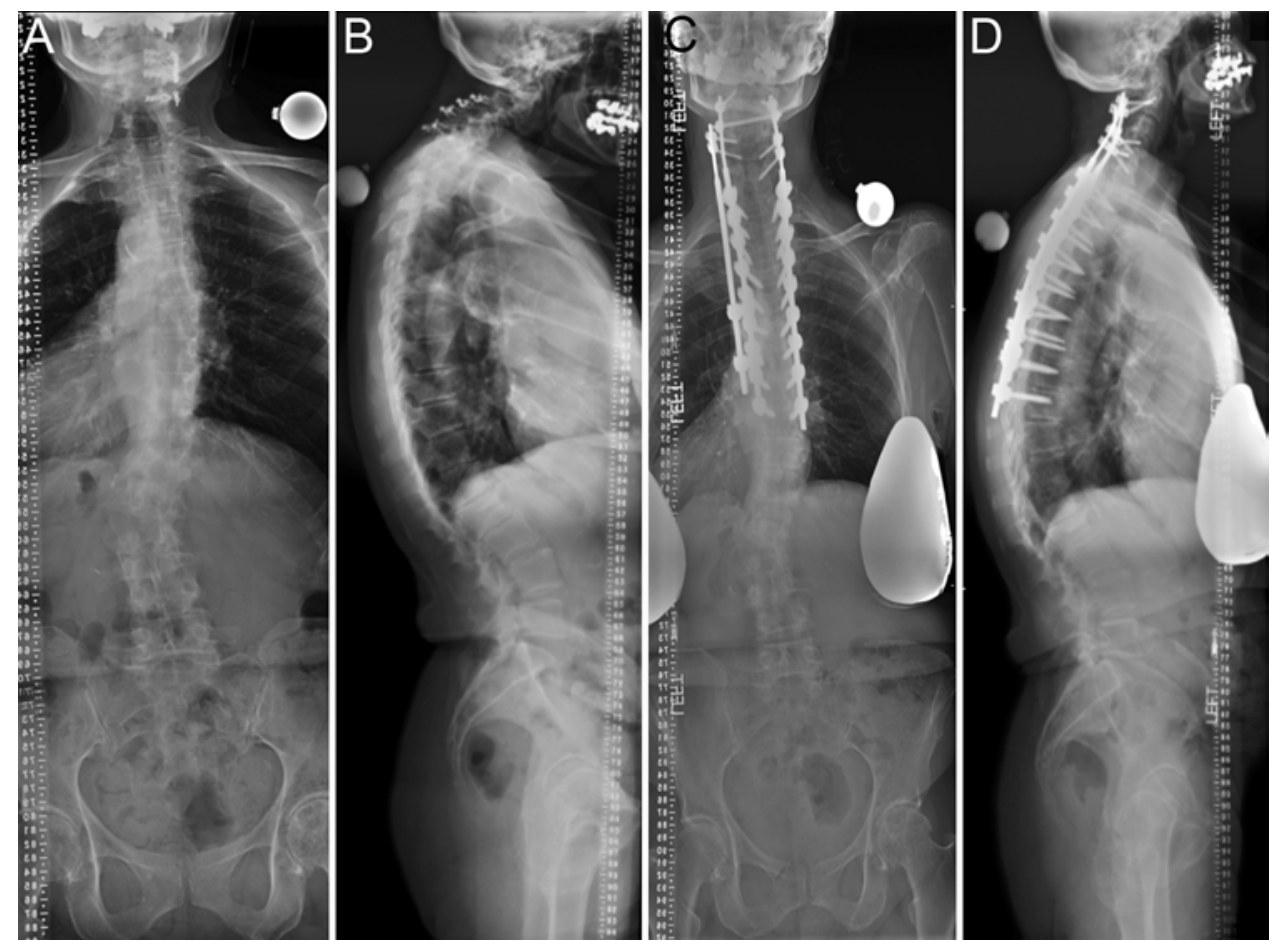

FIG. 2. Preoperative and postoperative scoliosis radiographs of a patient with severe cervical kyphosis who underwent deformity correction via C3-7 SPO and instrumented C2-T10 posterior spinal fusion. Prior cervical laminoplasty can be seen in her preoperative radiographs (A and $\mathbf{B}$ ). The patient's preoperative cervical parameters were cSVA, $7.7 \mathrm{~cm} ; \mathrm{CL},-40^{\circ}$ (kyphosis); $\mathrm{C} 2$ slope, $45^{\circ}$; and T1 slope, $37^{\circ}$. Her postoperative cervical parameters were cSVA, $5.6 \mathrm{~cm} ; \mathrm{CL}, 2^{\circ}$; $\mathrm{C} 2$ slope, $25^{\circ}$; and T1 slope, $34^{\circ}$ (C and D). 
it will be performed because this likely has a significant impact on neurological complication rates. As seen in this study, there were significantly lower neurological complication rates when $3 \mathrm{CO}$ was performed at more distal levels. This trend has been seen when comparing $3 \mathrm{CO}$ in the lower cervical region as opposed to the upper thoracic region, with lower cervical osteotomies associated with longer intensive care unit stay, hospital stay, and higher complications. ${ }^{26}$ As such, the senior author has made a transition to shift 3CO to the most distal vertebral levels when possible (balancing the risk of neurological complications with the ability to achieve adequate deformity correction). This may have contributed to nonsignificant differences in the surgical and neurological complication rates between $3 \mathrm{CO}$ and SPO.

As we continue to learn more about the clinical benefits of cervical deformity correction, it is imperative that we mitigate and minimize perioperative complications. In patients who have undergone cervical deformity correction, one of the most important predictors of worse Neck Disability Index and modified Japanese Orthopaedic Association scores at the 1-year follow-up was the occurrence of a major complication following surgery. ${ }^{34}$ Therefore, avoiding perioperative complications will in turn improve long-term outcomes for patients. According to the findings from our study, deformity severity seems to be an independent variable associated with risk for complications. Our study suggests that preoperative kyphosis $>20^{\circ}$ and cSVA $>8 \mathrm{~cm}$ are both independent risk factors for surgical complications. Kyphosis $>20^{\circ}$ was an independent risk factor for neurological complications as well. These findings are most likely because severe sagittal deformity requires greater corrective maneuvers. Therefore, preoperative counseling of patients regarding their increased risk of complication is important, and greater caution should be taken for such patients.

The main limitations of this study relate primarily to the retrospective nature of the data, relatively small cohort size, and heterogeneity of cervical deformity. Retrospective studies are at risk of inherent biases and unforeseen or unmeasurable confounders. We minimize heterogeneity between the two groups by studying a single-surgeon experience, but the best methodology of comparing two procedures would be to perform a multicenter prospective randomized trial. Severe cases of ACD are uncommon and make up a small fraction of a spine surgeon's practice, even for established deformity surgeons. Therefore, the number of study subjects is relatively small compared to other spine surgery studies. In this particular study, this limits the power of the analysis and constrains our ability to identify significant findings between smaller differences. An example includes the slightly higher neurological and surgical complication rates seen with $3 \mathrm{CO}$ compared to SPO, which were not significant on statistical analysis. Nonetheless, this is the largest single-center cohort study reported to date and it still has adequate power for multivariate analysis. It is also important to highlight that cervical deformity is a heterogeneous disease, and the surgical management varies based on numerous patients and deformity-specific factors. Patients who undergo SPO and 3CO likely have different types of cervical deformity, and this may limit the ability to directly compare complica- tions and outcomes between those patients. At the same time, it is critical to understand the complication profile and differences when discussing surgical options with patients and deciding whether multilevel SPO or $3 \mathrm{CO}$ is the best strategy in cases when both osteotomies are suitable.

\section{Conclusions}

Posterior-based multilevel SPO and 3CO are powerful techniques for the correction of moderate to severe cervical sagittal deformity. Our findings suggest that excellent and equivalent correction can be achieved with either technique, with the exception that SPO does not change T1 slope and that the extent of correction is influenced by the severity of the cervical deformity. Complication rates were high, with approximately $40 \%$ of patients experiencing at least one complication. The neurological, surgical, and medical complication rates were $16.8 \%, 17.9 \%$, and $23.2 \%$, respectively. Patients who underwent $3 \mathrm{CO}$ had higher rates of neurological and surgical complications; when compared to larger cohorts, we would expect to see a statically significant difference. Therefore, larger cohort studies are needed to further validate these findings. Independent risk factors for surgical complications identified in our analysis included male sex, preoperative kyphosis $>20^{\circ}$, and preoperative cSVA $>8 \mathrm{~cm}$. Preoperative kyphosis $>20^{\circ}$ was also a risk factor for neurological complications. Patients exhibiting these factors should be counseled preoperatively about their increased risk for complications. Unfortunately, these specific-risk factors are not modifiable. A combined anterior-posterior approach was an independent factor for medical complications. This suggests that when possible, a posterior-only approach may minimize medical-related complications. Additional studies are needed on this topic, especially multicenter prospective studies, in order to impact surgical decision-making for this population of patients.

\section{References}

1. Smith JS, Line B, Bess S, et al. The health impact of adult cervical deformity in patients presenting for surgical treatment: comparison to United States population norms and chronic disease states based on the EuroQuol-5 Dimensions questionnaire. Neurosurgery. 2017;80(5):716-725.

2. Griegel-Morris P, Larson K, Mueller-Klaus K, Oatis CA. Incidence of common postural abnormalities in the cervical, shoulder, and thoracic regions and their association with pain in two age groups of healthy subjects. Phys Ther. 1992;72(6):425-431.

3. Jarzem PF, Quance DR, Doyle DJ, et al. Spinal cord tissue pressure during spinal cord distraction in dogs. Spine (Phila Pa 1976). 1992;17(8)(suppl):S227-S234.

4. Iida H, Tachibana S. Spinal cord intramedullary pressure: direct cord traction test. Neurol Med Chir (Tokyo). 1995;35(2):75-77.

5. Shimizu K, Nakamura M, Nishikawa Y, et al. Spinal kyphosis causes demyelination and neuronal loss in the spinal cord: a new model of kyphotic deformity using juvenile Japanese small game fowls. Spine (Phila Pa 1976). 2005;30(21):23882392.

6. Chavanne A, Pettigrew DB, Holtz JR, et al. Spinal cord intramedullary pressure in cervical kyphotic deformity: a cadaveric study. Spine (Phila Pa 1976). 2011;36(20):1619-1626.

7. Scheer JK, Tang JA, Smith JS, et al. Cervical spine align- 
ment, sagittal deformity, and clinical implications: a review. $J$ Neurosurg Spine. 2013;19(2):141-159.

8. Deviren V, Scheer JK, Ames CP. Technique of cervicothoracic junction pedicle subtraction osteotomy for cervical sagittal imbalance: report of 11 cases. J Neurosurg Spine. 2011;15(2):174-181

9. Etame AB, Wang AC, Than KD, et al. Outcomes after surgery for cervical spine deformity: review of the literature. Neurosurg Focus. 2010;28(3):E14.

10. Kim HJ, Piyaskulkaew C, Riew KD. Comparison of Smith-Petersen osteotomy versus pedicle subtraction osteotomy versus anterior-posterior osteotomy types for the correction of cervical spine deformities. Spine (Phila Pa 1976). 2015;40(3):143-146.

11. Etame AB, Than KD, Wang AC, et al. Surgical management of symptomatic cervical or cervicothoracic kyphosis due to ankylosing spondylitis. Spine (Phila Pa 1976). 2008:33(16):E559-E564.

12. Smith JS, Ramchandran S, Lafage V, et al. Prospective multicenter assessment of early complication rates associated with adult cervical deformity surgery in 78 patients. Neurosurgery. 2016;79(3):378-388.

13. Ames CP, Smith JS, Scheer JK, et al. A standardized nomenclature for cervical spine soft-tissue release and osteotomy for deformity correction: clinical article. J Neurosurg Spine. 2013;19(3):269-278.

14. Tang JA, Scheer JK, Smith JS, et al. The impact of standing regional cervical sagittal alignment on outcomes in posterior cervical fusion surgery. Neurosurgery. 2012;71(3):662-669.

15. Guérin P, Obeid I, Gille O, et al. Sagittal alignment after single cervical disc arthroplasty. J Spinal Disord Tech. 2012;25(1):10-16.

16. Jagannathan J, Shaffrey CI, Oskouian RJ, et al. Radiographic and clinical outcomes following single-level anterior cervical discectomy and allograft fusion without plate placement or cervical collar. J Neurosurg Spine. 2008;8(5):420-428.

17. Villavicencio AT, Babuska JM, Ashton A, et al. Prospective, randomized, double-blind clinical study evaluating the correlation of clinical outcomes and cervical sagittal alignment. Neurosurgery. 2011;68(5):1309-1316.

18. Passias PG, Jalai CM, Lafage V, et al. Primary drivers of adult cervical deformity: prevalence, variations in presentation, and effect of surgical treatment strategies on early postoperative alignment. Neurosurgery. 2018;83(4):651-659.

19. Staub BN, Lafage R, Kim HJ, et al. Cervical mismatch: the normative value of T1 slope minus cervical lordosis and its ability to predict ideal cervical lordosis. J Neurosurg Spine. 2018;30(1):31-37.

20. Law WA. Osteotomy of the cervical spine. J Bone Joint Surg Br. 1959;41-B:640-641.

21. Urist MR. Osteotomy of the cervical spine; report of a case of ankylosing rheumatoid spondylitis. J Bone Joint Surg Am. 1958;40-A(4):833-843

22. Simmons EH. The surgical correction of flexion deformity of the cervical spine in ankylosing spondylitis. Clin Orthop Relat Res. 1972;86(86):132-143.

23. Chin KR, Ahn J. Controlled cervical extension osteotomy for ankylosing spondylitis utilizing the Jackson operating table: technical note. Spine (Phila Pa 1976). 2007;32(17):19261929.

24. Scheer JK, Tang JA, Buckley JM, et al. Biomechanical analysis of osteotomy type and rod diameter for treatment of cervicothoracic kyphosis. Spine (Phila Pa 1976). 2011;36(8):E519-E523.

25. Smith JS, Shaffrey CI, Lafage R, et al. Three-column osteotomy for correction of cervical and cervicothoracic deformities: alignment changes and early complications in a multicenter prospective series of 23 patients. Eur Spine $J$. 2017;26(8):2128-2137.
26. Theologis AA, Tabaraee E, Funao H, et al. Three-column osteotomies of the lower cervical and upper thoracic spine: comparison of early outcomes, radiographic parameters, and peri-operative complications in 48 patients. Eur Spine J. 2015;24(suppl 1):S23-S30.

27. Tobin MK, Birk DM, Rangwala SD, et al. T-1 pedicle subtraction osteotomy for the treatment of rigid cervical kyphotic deformity: report of 4 cases. J Neurosurg Spine. 2017;27(5):487-493.

28. Theologis AA, Bellevue KD, Qamirani E, et al. Asymmetric C7 pedicle subtraction osteotomy for correction of rigid cervical coronal imbalance secondary to post-traumatic heterotopic ossification: a case report, description of a novel surgical technique, and literature review. Eur Spine J. 2017;26(1) (suppl 1):141-145.

29. Wollowick AL, Kelly MP, Riew KD. Pedicle subtraction osteotomy in the cervical spine. Spine (Phila Pa 1976). 2012;37(5):E342-E348.

30. Kim HJ, Nemani VM, Daniel Riew K. Cervical osteotomies for neurological deformities. Eur Spine J. 2015;24(suppl 1):S16-S22.

31. Yuk FJ, Rasouli JJ, Arginteanu MS, et al. The case for T2 pedicle subtraction osteotomy in the surgical treatment of rigid cervicothoracic deformity. J Neurosurg Spine. 2019;32(2):248-257.

32. Samudrala S, Vaynman S, Thiayananthan T, et al. Cervicothoracic junction kyphosis: surgical reconstruction with pedicle subtraction osteotomy and Smith-Petersen osteotomy. Presented at the 2009 Joint Spine Section Meeting. Clinical article. J Neurosurg Spine. 2010;13(6):695-706.

33. Tokala DP, Lam KS, Freeman BJ, Webb JK. C7 decancellisation closing wedge osteotomy for the correction of fixed cervico-thoracic kyphosis. Eur Spine J. 2007;16(9):1471-1478.

34. Smith JS, Shaffrey CI, Kim HJ, et al. Comparison of best versus worst clinical outcomes for adult cervical deformity surgery. Global Spine J. 2019;9(3):303-314.

\section{Disclosures}

Dr. Deviren reports being a consultant for NuVasive, Zimmer Biomet, SeaSpine, Alphatec/Atec, and Medicrea; and receiving royalties from NuVasive. Dr. Ames reports being an employee of UCSF and a consultant for DePuy Synthes, Medtronic, Stryker, Medicrea, K2M, and Zimmer Biomet; receiving royalties from Stryker, Zimmer Biomet, DePuy Synthes, NuVasive, Next Orthosurgical, K2M, and Medicrea; receiving research support from Titan Spine, DePuy Synthes, and ISSG; being on the editorial board of Operative Neurosurgery; receiving grant funding from SRS; being on the executive committee of ISSG; and being the director of Global Spine Analytics.

\section{Author Contributions}

Conception and design: Lau, Ames. Acquisition of data: Lau. Analysis and interpretation of data: Lau, Deviren, Ames. Drafting the article: Lau. Critically revising the article: all authors. Reviewed submitted version of manuscript: all authors. Approved the final version of the manuscript on behalf of all authors: Lau. Statistical analysis: Lau. Study supervision: Ames.

\section{Correspondence}

Darryl Lau: University of California, San Francisco, CA. darryl. lau@ucsf.edu. 